ESJ Humanities

\title{
Entre unité et diversité linguistique : Quelle approche pour le Cameroun?
}

\author{
Ismaila Datidjo, Anthropologue \\ Claudin Karim Nana, Sociologue \\ Université de Dschang, Cameroun \\ Pierre François Edongo Ntede, Anthropologue \\ Université de Yaoundé I, Cameroun
}

Doi:10.19044/esj.2021.v17n28p127

Submitted: 19 August 2020

Accepted: 16 July 2021

Published: 31 August 2021
Copyright 2021 Author(s)

Under Creative Commons BY-NC-ND

4.0 OPEN ACCESS

Cite As:

Datidjo I., Nana C. K. \& Edongo Ntede P. F. (2021). Entre unité et diversité linguistique : Quelle approche pour le Cameroun ?. European Scientific Journal, ESJ, 17 (28), 127. https://doi.org/10.19044/esj.2021.v17n28p127

\section{Résumé}

Le Cameroun est considéré comme une Afrique en miniature. Cette caractéristique lui est attribuée du fait de sa diversité humaine faite de communautés culturelles quasi représentatives des composantes sociales disséminées à travers le continent noir. À en croire plusieurs sources écrites, il compte au-delà de 250 groupes ethniques et linguistiques dont la cohabitation est un fait. Toutefois, l'histoire du pays témoigne de temps en temps des ressentiments ou des heurts entre communautés voisines, toutes animées par une tendance à l'ethnocentrisme au point où il convient de se demander si la pluralité culturelle que connaît ce pays ne serait pas une entrave à sa bonne marche. Dans un contexte où des voix s'élèvent pour préconiser l'adoption d'une langue ou d'un corpus de langues pour en faire le verbe auxiliaire pour les Camerounais, alors même que les langues officielles que sont l'anglais et le français sont des objets de désaccord entre les concitoyens, une préoccupation à enjeux anthropologiques se dégage. Sacrifier la pluralité au profit d'une unité linguistique avec son caractère réducteur des richesses culturelles est-il envisageable, et à quel prix ? La réflexion que mène cet article explore les atouts et inconvénients de la diversité culturelle du Cameroun et la confronte à l'éventualité de réduire à un dénominateur commun, des expressions culturelles que le hasard des circonstances a rassemblées pour la construction du dessein national. À travers une démarche de type qualitatif et 
au moyen d'entretiens guidés réalisés auprès de trente individus provenant des dix régions du pays et appartenant à différents groupes ethniques, la perception qu'ont les Camerounais de diverses générations de la configuration multiculturelle de leur pays et des enjeux que celle-ci représente est questionnée. Par une approche déductive d'analyse, il s'est agi d'entrevoir ce à quoi un Cameroun réduit à une ou à quelques langues pourrait ressembler.

Mots clés : Unité linguistique, diversité culturelle, identité nationale, Cameroun

\title{
Between Unity and Linguistic Diversity: Which Approach for Cameroon?
}

\author{
Ismaila Datidjo, Anthropologue \\ Claudin Karim Nana, Sociologue \\ Université de Dschang, Cameroun \\ Pierre François Edongo Ntede, Anthropologue \\ Université de Yaoundé I, Cameroun
}

\begin{abstract}
Cameroon is considered a miniature Africa by many researchers and other thinkers. This characteristic is given due to its human diversity made up of cultural communities that are almost representative of the social components scattered throughout the black continent. According to several written sources, there are more than two hundred and fifty (250), ethnic and linguistic groups whose cohabitation remains a fact. However, from time to time, the history of the country bears witness to resentment or clashes between neighboring communities, all of which are driven by this very human impulse of ethnocentrism, to the point where we have to ask ourselves whether the cultural plurality of this country might not be an impediment to the smooth running of the nation. In a context where voices are being raised to advocate the adoption of a language or a corpus of languages as an auxiliary verb for Cameroonians, even though the official languages of English and French are the subject of disagreement among fellow citizens, a concern with anthropological stakes emerges. Is sacrificing plurality in favor of a linguistic unit with its reductive character of cultural wealth conceivable, and at what cost? This article explores the strengths and drawbacks of Cameroon's cultural diversity and confronts it with the possibility of reducing to a common denominator, cultural expressions that have been brought together by chance, for the construction of the national project. That is to say, through a qualitative approach and guided interviews conducted with thirty individuals spread
\end{abstract}


throughout the ten administrative regions of the country and identified from different ethnic groups, the perception of Cameroonians of various generations of the multicultural configuration of their country and the challenges it represents is investigated. Through a deductive approach of analysis, it is a question of glimpsing what a Cameroon reduced to one language or some languages could look like.

Keywords: Linguistic unity, cultural diversity, national identity, Cameroon

\section{Introduction}

Diversifié du point de vue de la culture, le Cameroun est un pays où cohabitent différentes communautés ethniques. Celles-ci sont disséminées sur l'ensemble du pays et connaissent un brassage culturel. Le mélange entre ces ethnies est tel qu'il devient de plus en plus difficile de rencontrer une famille ou un clan qui n'ait pas connu d'hybridation culturelle par l'intermédiaire de l'un de ses membres. Cet état de fait connote de la capacité qu'ont les différentes peuplades qui constituent cette nation à s'accepter et à vivre ensemble, un idéal auquel les pouvoirs publics aspirent pour promouvoir l'unité et l'intégration nationale. Sur la base des données empiriques, un paradoxe se dégage. Alors que la cohésion nationale met en évidence l'acceptation réciproque des différentes composantes culturelles du pays, de nombreuses velléités à connotation égoïstes persistent et concourent à saper la cohabitation entre des groupes ethniques réunis au sein de la même patrie par l'histoire. Cela voudrait dire que la cohabitation qui prévaut souffre de temps en temps d'insuffisances avérées.

Le processus d'unification du pays n'a pu taire jusque-là ni les querelles entre Anglophones et Francophones, ni prévenir les ambitions hégémoniques qui animent toutes les communautés nationales aujourd'hui. Les disparités entre Francophones et Anglophones, autant que les conflits interethniques altèrent le délicat climat de bonne intelligence qui prévaut entre les camerounais, car ils sont sources de querelles, de ressentiments et de heurts.

Alors même que les langues officielles héritées de la colonisation donnaient aussitôt à percevoir l'unité nationale bâtie autour d'une identité linguistique double incluant le français et l'anglais, la crise dite anglophone en cours donne à constater que ces deux locutions sont encore aujourd'hui de véritables dispositifs de querelles entre concitoyens. Dans le contexte social de la montée des idéologies à caractère communautaire et ethnocentrique, y compris des crises sociopolitiques multiformes que traverse le Cameroun moderne, des voix s'élèvent pour remettre en cause l'unité présupposée du pays, avec pour point d'ancrage l'élément linguistique, laquelle unité est fondée sur la double identité «français-et-anglais ». Dans ce sens, Tsofack 
(2002 : 24), rappelait déjà que : «en adoptant ainsi le français et l'anglais comme langues officielles, les autorités camerounaises de l'époque ont sans doute voulu résoudre le problème du plurilinguisme rampant dans le pays, afin de préserver l'unité nationale, dans une fédération encore fragile ».

Entre l'adoption d'une seule langue ou d'une collection de langues d'origines ethniques nationales comme langues officielles et le maintien de l'anglais et/ou du français qui sont eux-mêmes objets de désaccords entre concitoyens, aujourd'hui plus qu'hier, l'unité du Cameroun donne à penser qu'elle doit se construire sur le registre linguistique.

En partant du fait que la langue est un vecteur de diffusion et de consolidation de la culture (Sibille, 2002), la présente réflexion dégage un enjeu anthropologique porté sur les atouts et les déconvenues éventuelles de l'institutionnalisation d'un modèle linguistique qui, selon cette étude serait approprié à la construction de l'unité au Cameroun. Elle confronte ainsi la faisabilité d'un projet d'unité linguistique à la réalité d'un contexte ethnique et culturel pluriel et surtout très diversifié. Avec en plus du français et de l'anglais comme langues héritées de la colonisation, le Cameroun compte plus de 250 autres langues porteuses des cultures nationales. (Mukum Mbaku, 2005).

La méthodologie adoptée ici procède d'une approche qualitative. Au confluent des paradigmes du structuro-fonctionnalisme et du choc des cultures, une analyse lexico-sémantique réalisée à partir d'une revue documentaire et des données recueillies au moyen d'entretiens individuels semi-structurés, permet de mettre en évidence le sens que les Camerounais donnent à l'unité nationale du point de vue linguistique.

Une trentaine d'individus mobilisés sur la base de l'appartenance ethnique et linguistique constituent l'échantillon. Ces derniers retenus dans l'ensemble des dix régions administratives du Cameroun, sont représentatifs des aires culturelles identifiables de ce pays. La dialectique consiste d'abord à restituer la perception que ces répondants ont du multiculturalisme et de ses avantages et inconvénients. Ensuite, elle procède à l'analyse du problème de l'inter culturalité ou du mode de coexistence de diverses ethnies. Enfin, l'argumentaire s'emploie à ressortir des deux premières dimensions ainsi annoncées, la pertinence et les implications des schémas possibles de l'unification du Cameroun par les langues.

\section{Une esquisse de répartition du Cameroun en aires culturelles}

La partition du Cameroun en aires culturelles a longtemps fait l'objet de débats entre spécialistes en sciences anthropo-sociales, sans qu'il n'y ait de consensus sur une configuration particulière à adopter. Alors que pour les uns, une partition du Cameroun en trois aires serait plus convenable, pour les autres, une réparation en quatre, ou en cinq aires culturelles, serait appropriée. 
Mukum Mbaku (2005), par exemple, propose une répartition du Cameroun en cinq aires culturelles :

- les Hautes Terres de l'Ouest ou «Grass Fields» où se meuvent les peuplades Bamiléké, Bamoun, et d'autres petits groupes implantés dans la région du Nord-Ouest ;

- la Forêt Tropicale Côtière, peuplée de Bassa, de Douala, et de nombreuses autres ethnies habitant la région du Sud-Ouest ;

- la Forêt Tropicale du Sud, terroir des groupes béti, bulu, fang, et des pygmées reconnus par l'appellation « Bakas »;

- les régions Semi-arides du Nord, contrée des peuples islamisés dits du Sahel et des hautes terres centrales ;

- les milieux de Désert et des Hautes Terres Centrales habités par les Kirdis, certains peuples non islamisés et des ethnies récemment islamisés.

Cette division en cinq ensembles se rapportant à cinq aires culturelles, ne bénéficie pas de l'assentiment de tous. Une autre conception admet plutôt une partition du pays en quatre aires culturelles, ce qui remet en cause l'éventuelle division en trois ensembles, celle-ci faisant l'objet de critiques de la part de certains spécialistes des cultures, et des ethnies qui ne voient pas la pertinence de rapprocher des peuplades n'ayant aucune proximité. Ainsi, adopter une répartition en trois aires culturelles, c'est prendre le risque d'associer des communautés sans affinités. C'est aussi ignorer le facteur environnemental qui est déterminant dans la configuration des cultures.

En esquissant une répartition du Cameroun en quatre aires géographiques, la cartographie culturelle pourrait se présenter comme suit :

- le Grand-Nord sahélien, avec ses différentes communautés musulmanes et non musulmanes, qu'on peut estimer proches du fait des affinités culturelles, c'est-à-dire, une proximité d'habitudes et de comportements, de même que le partage de certaines réalités historiques ;

- le Grand-Ouest des hautes terres qui est majoritairement constitué de certains peuples dits «bantous » et « semi-bantous » repartis dans les régions de l'Ouest et du Nord-Ouest, avec une extension dans la partie supérieure de la région du Sud-Ouest. Cette étendue faite de montagnes et d'un climat frais, n'est pas sans incidence sur les modes de vie de ses populations ;

- l'aire culturelle du Grand-Sud de forêt, qui regroupe les peuples dits «bantous » répartis sur les régions du Centre, du Sud et de l'Est. La particularité de cette aire culturelle est indissociable de la forêt 
équatoriale qui amène ses habitants à développer une culture adaptée aux contraintes que leur impose un tel milieu ;

- l'aire géographique Littorale ou côtière, avec ses populations elles aussi « bantoues » certes, mais des Bantous vivant dans la zone côtière et ses environs. Le Littoral camerounais, entendu comme aire culturelle, implique la région du Littoral proprement dite, une partie de la région du Centre et une portion assez large de la région du SudOuest car, ces zones sont d'une particularité géophysique relativement identique qui donne aux populations riveraines de l'océan atlantique une ressemblance des traits culturels.

Carte :Une esquisse de décomposition du Cameroun en quatre aires culturelles

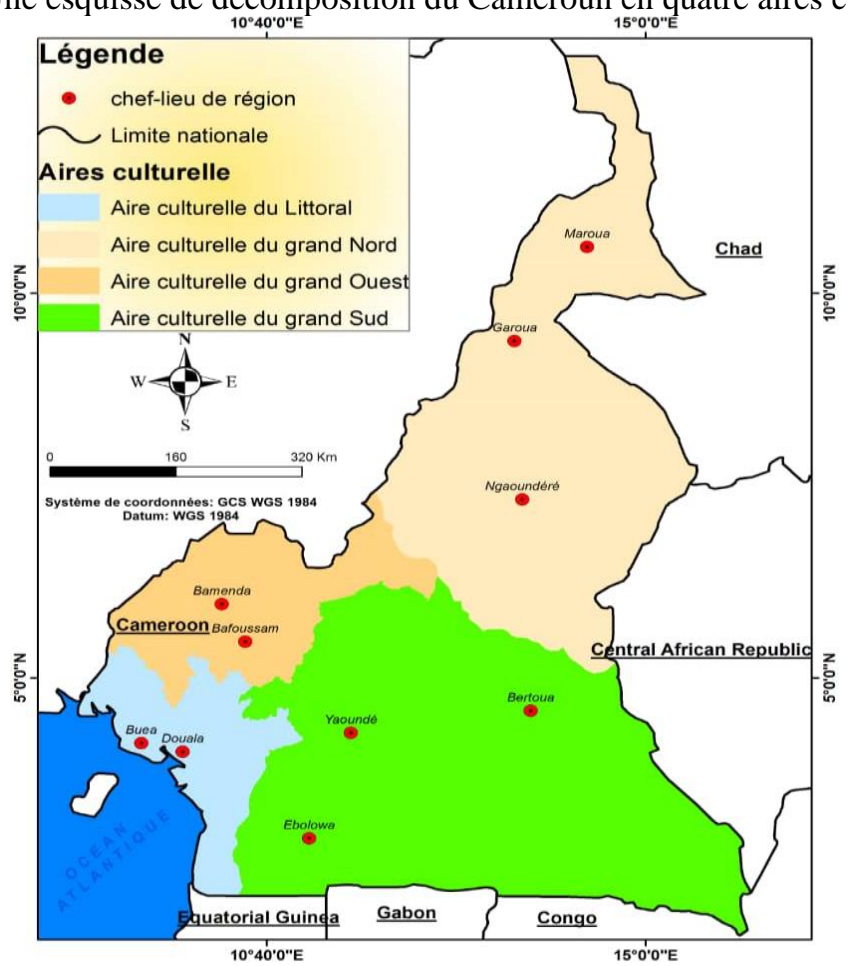

Auteurs : Ismaïla Datidjo et Nanfack

Cette carte illustrative de la répartition du Cameroun en quatre aires culturelles s'inspire non seulement de la composition ethnoculturelle de ce pays, mais aussi de sa diversité géophysique qui est indissociable des modes de vie des populations. Toutefois, il y a lieu de ne pas perdre de vue le fait que les frontières de ces aires culturelles ne sont pas en réalité hermétiques, étant 
donné qu'il existe des interpénétrations entre les composantes sociales qui s'y côtoient.

\section{Les enjeux du multiculturalisme au Cameroun}

Les avis des personnes que nous avons interrogées divergent quant à la perception du paysage multiculturel de leur pays. De manière générale, ils pensent que la multiplicité des ethnies peut être un avantage ou un désavantage pour le pays, selon les considérations qu'eux-mêmes ont de cette diversité de peuples et de cultures. À ce sujet, trois profils se dégagent, en fonction de leurs sensibilités au caractère multiculturel du pays.

\subsection{La perception du multiculturalisme et du multilinguisme}

Trois catégories de répondants se dégagent lorsqu'il s'agit de rendre compte du regard qu'ils ont du multiculturalisme et du multilinguisme impliquant à la fois les langues issues des cultures nationales et les langues officielles que sont le français et l'anglais désormais admis comme patrimoines nationaux.

\subsubsection{Le regard positivant du pluralisme linguistique arrimé au multiculturalisme}

Dans la catégorie des répondants favorables à la diversité culturelle de leur pays (17 sur 30 ; soit $56.66 \%$ ), le point de vue partagé est que la configuration actuelle du Cameroun est avant tout une aubaine, un atout. Il y a lieu de la préserver pour le bien de tous, car c'est en cela que devrait consister une République «des peuples ». Elle doit être multi faciale et multipolaire dans une approche systémique où les parties constituantes du même ensemble se tiennent et s'accompagnent pour la réalisation du dessein fondamental commun. Les tenants et défenseurs d'une image du Cameroun multiethnique et diversifié du point de vue linguistique mettent l'accent sur le potentiel économique que récèle le multiculturalisme et la pluralité ethnique comme gage d'une singularité de prestige.

\subsubsection{Le multiculturalisme comme potentiel économique}

Pour les personnes interrogées, la diversité culturelle du Cameroun est une source d'attraction, précisément pour les touristes étrangers. Elles pensent que le fait que ce pays soit composé de plusieurs cultures est une source d'attraction, et donc un facteur non négligeable de développement économique. Comme l'indique l'une d'entre elles: «la diversité culturelle enrichit le patrimoine national, constitue un attrait pour la curiosité du monde extérieur et favorise le tourisme culturel ». Dans le même sens, une autre pense 
que : « le patrimoine culturel national qui fait du Cameroun une Afrique en miniature est favorable à l'éclosion du tourisme ».

À en croire les informateurs, la diversité culturelle est un atout qui peut être capitalisé pour booster la rentabilité économique du pays, notamment grâce au développement du secteur touristique. Cela est un fait parce qu'à chaque peuple correspond une culture, c'est-à-dire un mode singulier de vie, avec tout ce qu'il implique comme art et potentiel de créativité. Ce regard correspond à une idée soutenue par Piccardo (2016), qui voit une relation positive entre le plurilinguisme et la créativité artistique. Elle pense, en effet, que grâce à sa nature multiple et flexible qui valorise toute forme d'hybridation et qui permet d'accroitre la prise de conscience de l'unicité de chaque trajectoire individuelle, le plurilinguisme peut créer des conditions favorables à la créativité. À son tour, la créativité, en tant que capacité transversale favorisant la flexibilité cognitive, constitue une (res)source puissante pour valoriser la diversité culturelle et le multilinguisme des sociétés.

De ce qui précède, il ressort que pluralité des langues et créativité seraient connectées l'une à l'autre selon une symétrie qui leur permettrait de maintenir leur existence commune en travaillant à développer la société multilingue. Ainsi, la différence serait à la base du comportement de créateur, d'inventeur et d'innovateur, surtout dans un contexte de compétitivité où cette différence suscite en chaque singularité, le désir de s'affirmer et de devancer ses concurrents. Dans le registre des concurrences interethniques, chaque communauté exprime sa fierté de disposer dans son terroir, des atouts dont l'exploitation constitue son moyen d'affirmation identitaire.

Les habitants des hautes terres de l'Ouest se réjouissent d'être de bons cultivateurs certes, mais cela du fait d'un climat et d'un sol favorable à l'agriculture. Les ressortissants des zones de savane et de steppe expriment, à leur manière, la fierté d'être de bons pasteurs et par extension, ingénieux pour exercer les travaux agricoles malgré le caractère aride et semi-aride de leur niche écologique. Les peuples des milieux forestiers se contentent d'avoir à longueur de l'année, une pluviométrie abondante qui leur accorde une aire humide et propice aux travaux champêtres, sources intarissables d'acquisition des biens alimentaires, et surtout un dispositif forestier dont l'exploitation constitue un poumon économique. Les populations des contrées de la côte sont satisfaites des avantages liés aux activités halieutiques et maritimes auxquelles s'ajoutent celles agricoles qu'ils exercent dans leur environnement. Si ces atouts énumérés ne sont que quelques constituants des richesses dont regorgent les aires géographiques substituées en aires culturelles du Cameroun, il y a aussi lieu de mentionner, selon certains informateurs, les richesses minières préexistantes et celles en cours d'exploration à travers le pays qui, mises ensemble, feraient une force économique considérable. Étant 
donné que ces répondants appartiennent à la la catégorie de ceux qui voient en la diversité des sociétés ethniques un potentiel économique profitable à tous, ils préconisent la détermination dans le travail et en appellent à une mise en valeur des savoir-faire ethniques. Ils prônent également une exploitation optimale des richesses que regorge chaque aire géographique à résonnance culturelle pour le bien de tous.

\subsubsection{La pluralité ethnique pour une singularité de prestige}

Avec plus de 250 groupes ethniques et culturels, chacun ayant sa spécificité linguistique, le Cameroun est, pour plusieurs informateurs, d'une caractéristique singulière. À ces langues nationales, il faut ajouter les langues officielles que sont l'anglais et le français, issus du passé colonial. La mosaïque culturelle et linguistique du Cameroun lui confère une identité qui le distingue de nombreux autres pays d'Afrique et du monde.

Dans le monde justement, le Cameroun est l'un des très rares pays à disposer du français et de l'anglais comme langues officielles. Cette caractéristique particulière, à laquelle s'identifient les Camerounais, fait en sorte que les gouvernements successifs de leur pays ne manquent pas d'évoquer la nécessité de les préserver. Pour y parvenir, disent-ils, il faut cultiver l'acceptation des différences et asseoir l'image d'une nation qui transcende son hétérogénéité culturelle au quotidien. Plus encore, ils pensent que c'est un prestige d'être unique en Afrique et de compter parmi les rares pays au monde à disposer de ces atouts ethnoculturels, tant chaque langue est porteuse de la culture qui l'a vu naître.

À propos de sa singularité, Tchawa (2012) pense que c'est l'histoire plus que tout autre facteur, qui donne au Cameroun son originalité, laquelle originalité le distingue des autres pays d'Afrique. En reprenant Ghalioun (1992), il affirme que : « la pluralité et les différences ne constituent nullement le ferment de la crise, mais plutôt une opportunité pour la cristallisation de la nation » (TchawA, 2012, p.320). Ainsi, cet auteur conçoit la diversité culturelle du Cameroun comme une aubaine. Au regard des réalités sur le terrain des interactions sociales, cette idée semble se rapporter à un idéal recherché, mais en contraste avec les données du vécu quotidien. Il se révèle qu'à certains égards, la diversité constituerait un facteur de désorganisation sociale et une menace pour l'intégrité nationale. Toutefois, il faut retenir que la catégorie sus-rapportée exprime clairement sa fierté d'être issue d'un pays singulier du fait de sa configuration ethnologique diversifiée. Pour cette catégorie de répondants, c'est une donnée extrêmement rare pour un pays africain d'avoir en son sein presque toutes les caractéristiques sociales de son 
continent. Ils y entrevoient une marque de prestige et une richesse culturelle pour laquelle il faut se réjouir et veiller à la préservation.

\subsection{Le repli identitaire ou la négation d'une identité plurielle du Cameroun}

D'un point de vue d'ensemble, les adversaires d'un Cameroun multiethnique et plurilinguistique (5 sur 30 ; soit $16.66 \%$ des informateurs), ont une posture de fermeture qui leur donne à voir les ethnies autres que la leur comme étant périphériques. De ce fait, ils font une catégorisation qui voudrait que leur culture d'appartenance incluant la langue ethnique, soit la référence, c'est-à-dire le centre autour duquel devraient pivoter les autres communautés linguistiques nationales. Suivant cette approche, ils valorisent leur appartenance qu'ils n'entendent pas assimiler, ni rapprocher d'une autre. Ils admettent la différence certes, mais une différence qui nie l'altérité et lui refuse toute considération. Pour ces informateurs, la préservation des identités culturelles singulières dans un contexte multiculturel est nécessaire, surtout quand les ethnies sont sans cesse en compétition du fait d'un jeu politique mal assimilé, où le militantisme d'opinion se confond et s'accommode avec la quête des intérêts ethniques. Dans les aires culturelles identifiées du Cameroun, il se développe une compétition entre les communautés voisines, chaque groupe ethnique cherchant à s'affirmer, voire à dominer les autres dans une espèce de course à l'hégémonie. Pourtant, cette compétition s'étend audelà des aires culturelles pour se manifester à une échelle plus grande qui met en rivalité l'ensemble des ethnies nationales et parfois les contrées, qui peuvent se rapporter soit aux aires culturelles, soit aux régions en tant que circonscriptions administratives.

Pour les tenants du repli identitaire, il y aurait un déséquilibre dans la considération que l'État accorde aux ethnies, étant donné que certaines d'entre elles seraient plus favorisées que d'autres. Aussi, au risque de voir les ethnies les plus en vue dominer, et peut-être assimiler les moins importantes et les moins considérées, ces tenants du repli identitaire pensent qu'il y a lieu d'éviter la communion des peuples.

La coexistence de différentes cultures permet de «découvrir sa personnalité à travers et par l'autre » (Hamidou, 2007, p.29). Malgré le fait que la différence soit le canal par lequel, en comprenant l'autre, une société cultive une meilleure connaissance d'elle-même, de nombreux répondants fustigent le rapport que les membres de leurs communautés entretiennent avec les personnes d'autres groupes ethniques. Ils vilipendent l'ouverture à l'altérité tout en ignorant l'idée de nation pour laquelle la différence reste un facteur fondamental. Toutefois, il convient de ne pas perdre de vue que l'appel au repli communautaire n'exclut pas l'existence au Cameroun, de l'ouverture et de la communion des ethnies. Cette ouverture permet mieux que toute autre 
attitude, ce à travers des interactions impliquant les personnes de contrées et cultures différentes, d'assurer la cohésion nationale, favorable à l'essor économique du pays.

Je prends un exemple; chez les gens du Noun (les Bamun), si tu épouses une femme, la famille de la femme prend la peine de quitter la maison de la fille qu'elle envoie en mariage. Maintenant si un Bamun veut épouser une Bamiléké, il va croire que la dot se passe comme chez eux où il y'a entente entre les deux familles avant de gérer le reste après. Pourtant, chez les Bamiléké la dot n'est pas doucement. S'il faut aller selon les normes, tu peux verser la dot d'une fille au minimum à un million et la famille n'a rien à t'offrir ; c'est toi qui a tout à donner. Pour les uns c'est compliqué et irraisonnable alors que pour les autres c'est normal. [...] Si on va à Douala, nous les Sawas, nous avons une façon de nous habiller en nouant le pagne autour de la hanche et en portant une chemise et une belle paire de babouches aux pieds; comme ça, nous sommes habillés de manière honorable et nous aimons bien ça. Pourtant quelqu'un d'ailleurs ne verrait pas ça d'un bon æeil...

Ce récit comme celui d'autres personnes atteste de l'attachement que les Camerounais ont pour leurs valeurs culturelles ethniques. Il contraste avec la conception qui voudrait que la différence soit un facteur constitutif de la nation avec laquelle ils doivent conjuguer.

De ce qui précède, il faut retenir que les «incubes» identitaires préconisent le repli et donc le rejet ou la négation de l'altérité ; ceux-là ne promeuvent pas une identité nationale inclusive qui serait la fédération des identités ethniques reflétant un type de Camerounais qui, malgré leurs particularités ethniques et linguistiques, font partie de la mosaïque des cultures que compte leur pays. Ils prônent plutôt des identités singulières se rapportant pour chacun à son groupe ethnique d'appartenance et repoussant de surcroît les autres dans leurs propres cocons ethniques et culturels.

\subsubsection{Les indécis du modèle linguistique : l'embarras du choix identitaire}

Sur la question de l'éventualité de réduire le système linguistique camerounais à une ou quelques langues nationales, une composante des enquêtés ( 8 sur 30 ; soit $26.66 \%$ des informateurs), expriment leur indécision sur la question. Ils naviguent à la fois entre les aspects positifs et les conséquences négatives qui découleraient de la réduction de la diversité en une unité linguistique ou en une diversité très limitée à un petit nombre de langues. Pour eux, autant un tel processus serait une avancée pour le pays, autant à cause de ses implications, il porterait en lui-même les germes de la 
désintégration de l'unité nationale. C'est ce que révèlent les propos recueillis chez ces derniers. D'après certains d'entre eux, le fait qu'une seule langue ou un corpus de quelques langues nationales soit adopté pour revêtir un caractère officiel aurait des conséquences à la fois positives et négatives. Les premières se rapportent au fait que chaque Camerounais, en dehors de sa langue maternelle, pourrait enrichir son bagage linguistique en apprenant d'autres langues. Les secondes ont trait au fait que les camerounais qui parlent les langues dites minoritaires et ceux dont la langue ethnique n'est pas adoptée pourraient ressentir de la frustration en y voyant le manque de considération pour leur groupe d'appartenance. Ceci ne peut qu'accentuer le tribalisme préexistant, qui est à l'origine de querelles entre concitoyens de différentes ethnies. Les autres conséquences sont relatives au fait que du point de vue supra national, ces indécis du modèle linguistique admettent que l'adoption d'une ou de quelques langues nationales comme langues officielles pourrait provoquer un isolement linguistique du pays, étant donné que ses relations multilatérales avec les autres nations en prendraient un coup. Rejeter le français ou l'anglais et éventuellement les deux, impliquerait de façon absolue un recommencement, alors que tout ce qui est institutionnel l'est à partir de ces deux langues. Il faudrait, en effet, traduire du français ou de l'anglais, tout texte institutionnel pour l'adapter au(x) nouveau(x) système(s) d'expressions à adopter et procéder de surcroît à une retranscription.

En plus de ces hypothèses visiblement contraignantes, d'autres indécis entrevoient l'émergence des difficultés communicationnelles non seulement entre les concitoyens dont le plus grand nombre doit se mettre à l'école de « la nouvelle langue », mais aussi, des difficultés relationnelles qui affecteraient la diplomatie et toutes les autres formes de rapprochement entre le pays, ses habitants et le reste du monde. Si telle est l'opinion d'une fraction d'enquêtés, leur position est similaire à celle d'autres répondants qui pensent que les conséquences liées à l'adoption d'une langue nationale comme officielle peuvent s'avérer positives, mais il ne faut pas pour autant négliger la portée négative que cela peut avoir.

L'option d'une langue ethnique nationale officialisée ou de quelques langues choisies dans les aires culturelles du pays, peut être positive si on y parvient. Cela est d'autant clair dans la mesure où cette langue, ou ces langues ainsi identifiées serviraient de moyens pour rapprocher les populations et les rassembler autour des idéaux nationaux. Les tenants de cette approche affirment que la culture est le moyen le plus sûr de lier les identités singulières en une seule, ou en une qui soit moins fracturée. Ils y voient la possibilité de renforcer l'unité nationale déjà mise à mal par la grande diversité ethnique qui 
fait des exclus ou des marginalisés, lesquels peinent à contenir leur frustration devant les groupes qui, selon eux, seraient privilégiés.

Au bout du compte, ceux qui se montrent plutôt perplexes, manquent d'arguments totalement en faveur ou contre le pluralisme culturel du pays. Pour eux, à la question de savoir, quelle unique approche préférez-vous entre le pluralisme linguistique en place et la réduction en une unité linguistique, ou en un corpus de quelques langues nationales, le verbe officiel des Camerounais, la réponse s'est plutôt voulue fuyante en général. Il ressort de l'analyse faite à ce propos que, ce qui importe pour la plupart des personnes rangées dans cette catégorie, c'est de vivre en toute quiétude et de vaquer à leurs occupations.

Le tableau ci-après résume l'analyse qui découle de la perception du multiculturalisme et du multilinguisme par les personnes interrogés.

Tableau : Récapitulatif des informateurs et positions par rapport au multilinguisme

\begin{tabular}{|c|c|c|c|c|}
\hline & $\begin{array}{c}\text { Favorables au } \\
\text { multilinguisme }\end{array}$ & $\begin{array}{c}\text { Défavorables au } \\
\text { multilinguisme }\end{array}$ & Indécis & Totaux \\
\hline Effectifs & 17 & 5 & 8 & 30 \\
\hline Taux (\%) & 567.66 & 16.66 & 26.66 & 100 \\
\hline
\end{tabular}

Source : Enquêtes de terrain

\section{Discussions : la diversité des cultures et langues, un sujet de controverses}

À la préoccupation de savoir dans quelle mesure peut-on sacrifier la pluralité au profit d'une unité linguistique avec son caractère réducteur des richesses culturelles au Cameroun, les points de vue sont divergents au sein d'une opinion nationale partagée, car confrontée à la pluralité linguistique importante et indissociée de la diversité ethnique et culturelle en place dans ce pays. Aussi, à la nécessité d'apprécier la faisabilité d'un tel dessein et d'en supputer les conséquences éventuelles, le présent article confronte les arguments recueillis et les analyse suivant une approche déductive qui conjecture sur le modèle qu'il convient d'adopter et ses atouts.

\subsection{La diversité culturelle, une gangrène pour l'unité nationale au Cameroun}

Pour des répondants, loin d'être une valeur, la diversité culturelle est une gangrène pour l'unité nationale. Elle est responsable, pour une bonne part, des déséquilibres sociaux que connait ce pays. Entre les discriminations et le favoritisme ethno-tribal et régional, le multiculturalisme en contexte camerounais serait, outre ses avantages, un mal pour le pays. En fonction de leurs contrées d'appartenance ou de provenance, ces enquêtés vilipendent le sentiment national au profit du communautarisme tribal et régional. Dans un tel contexte, les citoyens ne bénéficient pas d'égales opportunités, et la 
frustration des uns devient conséquemment la cause des ressentiments envers les autres.

\subsection{De la diversité culturelle aux marginalisés sociaux}

Nombreux sont les répondants qui pensent que le multiculturalisme est à l'origine des comportements discriminatoires observés çà et là dans leur vécu quotidien. Pour les uns, il est à la fois générateur du tribalisme, du favoritisme et des discriminations sociales. Selon un enseignant par exemple :

Dans le milieu professionnel déjà, on peut remarquer la corruption ; on peut aussi remarquer comment le tribalisme est l'une des préoccupations les plus prégnantes de la société camerounaise, parce que le problème est à la fois au niveau de la langue que vous parlez et de votre appartenance régionale. Tu vois, généralement quand un demandeur d'emploi vient à un boss, la première des choses à faire par celui-ci, c'est de trouver son nom, non pas pour une connaissance constructive, mais pour identifier son ethnie et sa contrée de provenance. L'attention est accordée à son dossier de candidature suivant la proximité entre son ethnie de provenance et celle du propriétaire de l'entreprise.

Dans le même sens, un autre enquêté pense que :

La pluralité culturelle et linguistique est un énorme obstacle pour le Cameroun, car au lieu de penser pour l' "ensemble Cameroun», les uns et les autres pensent pour leurs communautés culturelles, et l'État encourage cela à travers ce qu'il appelle "équilibre régional " lors des concours et autres recrutements dans la sphère administrative publique. La performance et le mérite sont relégués au second plan, le privilège étant accordé aux appartenances culturelles, ethniques et régionales. Ce qui encourage d'ailleurs les communautés à se constituer en groupes de pression pour réclamer à travers la pratique répandue de lettres ouvertes et mémorandums revendicatifs adressés aux instances publiques et personnalités de haut rang. Il s'agit par-là, de faire état de la situation "défavorable " d'une région ou d'une communauté particulière pour attendre des autorités en charge des prises de décisions, une amélioration des conditions décriées qui donnerait à ladite communauté ou région, une place privilégiée dans le « gâteau national » à partager.

Les propos de ces informateurs illustrent le fait que pour certains Camerounais, la pluralité des cultures est un facteur d'exclusion à la fois des personnes à une dimension réduite, et des communautés à une échelle plus étendue. Les marginalisations qu'elle crée entraineraient la situation de 
différenciation sociale entre les personnes et entre les groupes avec en extension, des comportements de favoritisme pour les proches et d'égoïsme envers ceux considérés comme différents de soi. Dans une telle situation, la langue est un facteur d'éligibilité dans les sphères professionnelles au mépris des compétences. Au-delà, la diversité culturelle devient le socle sur lequel se construisent l'idéologie et la posture de fermeture vis-à-vis des autres et de leur rejet systématique. De même, les particularismes qui sont les moteurs des rancœurs et des controverses interculturelles y puisent leurs sources ; ce qui consolide le paradigme de «l'ethnie-valeur suprême », qui a pour corollaire l'ethnocentrisme. À ce niveau, la diversité linguistique et donc culturelle du Cameroun, porte avec elle les germes de la désintégration de l'unité nationale, alors qu'elle devrait avoir pour fonction d'introduire une diversité constructive dans la société (Sibille, 2002). La diversité linguistique mettrait ainsi à mal l'unité du Cameroun, en ceci que la stratification linguistique entraine, à bien des égards, les replis identitaires et l'émergence des logiques ségrégationnistes. C'est dans ce sens que Tchinda Kenfo (2018), pense qu' « il reste difficile pour les Camerounais de dépasser leurs différences pour construire une nation multiculturelle débarrassée de luttes politiques et d'autres instrumentalisations ethniques et linguistiques ». (p. 1)

À la suite de cet auteur, il faut relever le danger que représente la diversité culturelle, dans la mesure où elle est soumise à des manœuvres de récupération qui l'instrumentalisent à des fins égoïstes. Elle ouvre de ce fait le champ à la construction du capital ethno-identitaire qui ne profite pas à la cause nationale. En plus d'être un obstacle à l'unité nationale, la diversité linguistique et culturelle serait aussi un frein au développement.

\subsection{La diversité linguistique, un frein au développement du Cameroun}

Pour plusieurs Camerounais, au-delà de l'unité nationale qui est mise à mal par la diversité culturelle et linguistique conséquente, le développement national subit lui aussi, les impacts négatifs inhérents de cette réalité. Au Cameroun, au lieu de se compléter pour la construction nationale, les ethnies et les cultures rivalisent. Loin d'une véritable acceptation réciproque des peuples et des langues qu'ils parlent, la réalité fait état des ressentiments des uns contre les autres dans un climat social de compétition entre les communautés constituées, tantôt suivant les aires culturelles, tantôt selon les régions administratives ou encore en fonction des ethnies. De ce fait, les uns minimisent les autres en accordant plus de valeur à leur culture et réciproquement, cela sur la base de la dichotomie centre et périphérie ou supériorité et infériorité. Les multiples affrontements intercommunautaires enregistrés au cours de l'histoire du Cameroun, à l'image des conflits Arabe Choa - kotoko, foulbé - Gbaya, ou Bamiléké - Mbo’o etc., sont des illustrations 
qui montrent que des ethnies qui ne se tolèrent pas n'acceptent pas réciproquement leurs langues.

Un autre regard «pessimiste» sur la pluralité linguistique du Cameroun perçoit cette réalité comme un obstacle pour le développement dans le sens où des phénomènes tels que le tribalisme ou le népotisme y sont courants. Dans ce sens, des informateurs pensent que la pluralité culturelle et linguistique constitue un obstacle pour le développement du Cameroun en tant que cause du tribalisme, du favoritisme et des discriminations. Ces différents points de vue mettent en évidence les raisons pour lesquelles le multiculturalisme, arrimé à la diversité des langues, serait un obstacle au développement. Ainsi, si le développement est un idéal pour toutes les communautés qui inclue prioritairement des libertés individuelles et collectives, alors le tribalisme, la corruption, et les autres fléaux sociaux résultant de la diversité culturelle en sont des freins.

L'imaginaire social camerounais s'est longtemps nourri de l'idée que certaines cultures prédisposent plus que d'autres au travail. Cette représentation parfois érigée en règle, contraste avec le sens du développement qui envisage la diversité comme richesse. C'est dans cet ordre de pensée qu'HENRY (2008) démontre que les acteurs sociaux interprètent toujours les situations auxquelles ils sont confrontés dans un contexte multiculturel où ils sont tous soumis aux mêmes contraintes. Cela dit, et à contrario, dans un contexte d'homogénéité culturelle, les acteurs sont tenus par la routine de leurs manières de faire communes et de leurs croyances, qui annihile leurs esprits d'innovation. Si les conclusions auxquelles aboutit cet auteur corroborent les points de vue des informateurs, ce serait justement parce que ce n'est pas la différence qui fait problème, mais le fait qu'elle soit instrumentalisée à des fins contre-productives. En contexte camerounais, le multiculturalisme ne serait donc pas un facteur de performance économique, mais plutôt, le socle sur lequel se construit l'ethnocentrisme.

\subsection{L'idée d'une répartition basée sur de grands groupes linguistiques}

Pour certains enquêtés, une configuration basée sur les "grandes composantes linguistiques »du pays serait une astuce pour rapprocher les Camerounais du point de vue de la langue. Cette approche consiste à identifier les langues locales les plus usitées pour en faire des langues officielles. Il s'agit de construire deux, trois ou quatre systèmes lexicaux qui permettront aux Camerounais de communiquer entre eux à partir des langues locales. Cette idée est nourrie par le fait que dans l'imaginaire de certains, il existe des langues plus diffuses que l'on peut reconnaitre tantôt grâce à leurs audiences, tantôt grâce au fait qu'elles soient liées à des ethnies dont les populations sont numériquement plus importantes et géographiquement plus disséminées. En effet, comme le pense un informateur, « il y a de grands foyers linguistiques 
au Cameroun ». Ce propos fait allusion notamment à l'aire culturelle constituée des ethnies des trois régions septentrionales et se réfère précisément à la langue foulbé auxiliaire dans cette partie du pays. Si dans cette étendue géographique le fulfulde se démarque de bien d'autres langues du terroir, il faut reconnaitre que dans le reste du pays, aucune autre langue ne se démarque de façon notoire des locutions voisines, ni ne dispose d'une audience assez répandue qui soit convaincante pour être admise comme langue dominante.

L'aire culturelle des Grass Fields ou des Hautes Terres de l'Ouest dont la composante ethnique bamiléké représente la plus grande, ne dispose pas d'une langue auxiliaire qui fasse l'unanimité car, malgré son importance numérique, le groupe ethnique bamiléké est un ensemble morcelé en une multitude de sous-groupes ayant chacun sa langue au point qu'il n'existe parfois aucune intercompréhension entre les différents membres. Toutefois, le Pidgin en tant que sociolecte né d'un assemblage de plusieurs expressions incluant celle des langues ethniques nationales et surtout de la langue officielle qu'est l'anglais, dispose d'une large audience qui dépasse son contexte spatial d'élaboration, le terroir anglophone, faisant de surcroît partie de l'aire culturelle des Hautes Terres de l'Ouest. Ce pidgin est favorablement vu par certains répondants comme une langue dont l'adoption en tant que verbe national serait intéressante. En ne faisant pas l'unanimité, le Pidgin camerounais compte d'innombrables adversaires qui lui reprochent tantôt son non-appartenance à une communauté ethnique du pays, tantôt son absence de règles et l'incursion continue des nouveaux mots sans aucune régulation. Aussi, il lui est reproché par les francophones, son importante imprégnation d'expressions anglaises transformées et «tropicalisées » qui perdent parfois de leurs sens originels. À travers la négation du Pidgin camerounais, les Francophones expriment du dédain pour cette langue, et par extension le refus de voir l'anglais choisi comme seule langue officielle. Leur position traduit aussi celle des Anglophones envers le français qu'ils n'admettent ni ne considèrent au même titre que l'anglais.

\subsection{Le multiculturalisme : entre intégration et désintégration nationale}

Qu'elle soit perçue négativement ou positivement, la diversité culturelle reste un fait, une réalité au Cameroun. S'il est vrai qu'ils la vivent et qu'ils l'ont toujours vécue, les Camerounais dans leur ensemble ne s'accordent pas quant à l'attention portée à cette réalité, tant il prévaut des contradictions qui traduisent en conséquence les perceptions que les uns et les autres se font du multiculturalisme et, partant, de la diversité linguistique qui l'accompagne indissociablement. Des avis recueillis auprès des enquêtés confirment cet état de fait. En effet, certains d'entre eux affirment que ce qui divise les Camerounais au sujet de la diversité culturelle, c'est justement la 
diversité elle-même. Elle est la source de nombreux problèmes, surtout du tribalisme qui affecte pratiquement toutes les dimensions de la vie quotidienne. Par contre, ce qui les unit et donne l'image de la concorde et du « vivre ensemble » actuel, ce sont plutôt les préoccupations quotidiennes de survie. Elles transcendent les querelles interethniques et attitudes ethnocentriques existantes.

Pour d'autres répondants, ce qui divise les Camerounais c'est la pluralité ou diversité des cultures, et ce qui les unit, c'est l'appartenance à la même nation, le Cameroun. Parlant de la diversité culturelle, des informateurs considèrent l'ethnocentrisme comme étant à l'origine de la désunion des Camerounais, car la fierté affirmée et la supériorité auto proclamée de certains groupes ethniques sur d'autres ne font qu'attiser les brouilles entre concitoyens. En abondant dans le même sens, une opinion supplémentaire atteste que la désunion des Camerounais provient non seulement du fait que chacun voudrait que sa culture soit mise en l'avant, au détriment de celle des autres, mais aussi et pour une part importante, du fait que les uns développent des préconceptions pour dénigrer les autres et réciproquement. Il ressort de ce qui précède que chez les Camerounais interrogés, l'attention accordée à la diversité ethnolinguistique est fonction du sens qu'elle revêt pour les individus et les groupes auxquels ils appartiennent et des implications qui en découlent.

\subsection{La diversité, un problème à la base}

Chez de nombreux enquêtés (donner le nombre et le pourcentage), la diversité, au-delà de ses implications, est en elle-même un problèmePour ces derniers, tout se passe comme si dans un contexte de non diversité, il y aurait concorde sociale et communion des ethnies. Ainsi, la diversité culturelle est comprise comme le germe de la désorganisation sociale. Cette manière de penser pourrait justifier la montée des revendications nationalistes qui visent la promotion de l'unité nationale à partir des valeurs communes. Pour cette idéologie du nationalisme, la diversité des ethnies et des langues rime avec la division. La vision d'un Cameroun « uni » renvoie ici à l'idée d'une identité linguistique qui permettrait de résister à toute ingérence étrangère, notamment celle des anciennes métropoles colonisatrices. La remarque qui peut être faite ici est que l'unité linguistique va avec l'unité culturelle ; au Cameroun, elle semble être chimérique.

Dans le contexte actuel de mondialisation où les langues participent des rapports de pouvoir (Pilote et al., 2010), les problèmes de l'identité sociale sont là. Certains ont surinvesti leurs sentiments et passions dans l'intégrisme ethnique, national ou religieux, où se mêlent et se cachent souvent d'autres intérêts particuliers, dans un engagement figé qui les mènent vers l'agression (Laburthe-Tolra, 2010). À en croire cet auteur, en situation de multiculturalité, soit on oppresse, soit on est oppressé. Il n'y a donc pas de neutralité, mais la 
neutralisation de l'autre. Subséquemment, le sens que prennent ici les notions de tribalisme et de favoritisme est stratégique et induit l'idée de l'imperméabilité des groupes sociaux. La diversité culturelle serait alors un élément facilitateur pour la construction des inégalités sociales.

\subsection{La neutralité du français et de l'anglais profitable à l'unité nationale}

Au-delà du péril de la diversité culturelle au Cameroun, le sentiment d'appartenir à une même nation devrait être le soubassement de la communion des ethnies et de l'unité du pays. À ce niveau, le consensus d'unité nationale ne saurait être la négation des identités spécifiques, il doit plutôt exprimer la capacité des personnes et des groupes, à transcender la différence pour considérer ce qui réunit. Dans ce sens, le français et l'anglais en tant que langues officielles doivent être des facteurs de cohésion et d'intégration nationale. Il faut les préserver comme telles. Leur caractère de langues empruntées dissociées de toute filiation ethnique marque leur neutralité et fait d'elles, des atouts pour la construction et la consolidation de l'unité nationale.

«La langue est d'un enjeu capital dans la construction de la cohésion sociale. Elle est ce qui anime et détermine les rapports entre les individus » (Abia Aboa, 2012, p.10). En s'inspirant de ce qui précède, et en contexte camerounais où les ethnies et les langues se livrent à des concurrences en générant continuellement des rancœurs, on peut poser sans absolutisme, le français et l'anglais comme arbitres du fait de leur neutralité de départ. Il est évident que la composition du pays en deux parties ayant respectivement et prioritairement l'une ou l'autre langue en usage, traduit en soi des oppositions, car ces langues ont été adoptées par les ethnies qui y résident. Vis-à-vis de cet état de fait, il y a lieu de consolider l'acquis et de dépasser les considérations actuelles en octroyant à ces langues, au moins au niveau institutionnel et sur toute l'étendue du pays, la même place. Il s'agit à cet effet d'enclencher une révolution du système éducatif national qui inclut l'utilisation concomitante des deux verbes sans exclusivité dès la base, avec en vue des résultats pérennes à envisager sur le moyen ou le long terme.

\subsection{L'utopie de l'unité linguistique au Cameroun}

L'oralité' est la marque du génie africain et le gage du développement comme système de production réfléchi, mûri et adopté par une communauté linguistique. Le procès fait à l'Afrique de refuser le développement porte à la fois sur le rejet des langues africaines et l'adoption de celles des colonisateurs. Pourtant, ignorer la langue de ses ancêtres équivaut à perdre ses racines culturelles (Tchindjang et al., 2008). Cette pensée dévoile le nœud du problème linguistique en Afrique en général et au Cameroun en particulier. Faut-il délaisser les langues importées pour valoriser les langues locales ? 
Cette interrogation induit de penser la possibilité de réduire la diversité des langues du Cameroun à une unique, qui soit propre à ce terroir et commune à toutes ses composantes culturelles. À ce propos, les avis des personnes interrogés convergent. Il serait irréaliste de faire aboutir un projet d'unification linguistique. Les prises de position en sont illustratives. Cela dit, le fait que les deux langues officielles actuelles soient des sujets de désaccords connote à suffisance de la difficulté de fédérer la diversité linguistique en une seule unité. Un informateur explique :

À mon avis, s'il faut remplacer toutes les langues du pays par une seule, ça deviendrait un problème. S'il faut choisir entre une langue du pays et les deux langues officielles actuelles, je dis déjà que les deux langues officielles reconnues par l'administration posent problème. Le pays est en train de se diviser; regarde la guerre dans le Nord-Ouest et le Sud-Ouest. Si des langues étrangères posent problème, qu'en sera-t-il de la langue bassa, toupouri, ou éton... qu'est-ce que j'en sais ? Comment peut-on opérer un choix dans près de 300 langues? S'il faut donner mon avis, alors je préfère encore qu'on s'en tienne aux langues officielles et ça serait mieux.

Dans le même sens, un autre informateur affirme :

Je pense qu'il n'est pas possible de remplacer nos langues officielles par une ou plusieurs langues nationales, car au Cameroun il existe plus de 250 ethnies; de ce fait je pense qu'il serait difficile de déterminer quelle seraient la langue ou les langues nationales qui pourraient remplacer les langues officielles. En plus, que deviendraient le français et l'anglais? Puisqu'elles sont notre héritage de la colonisation, elles font partie de notre histoire. Notre système éducatif, et tous les autres aspects de la vie en sont dépendants. Ce pays a été bâti avec le français et l'anglais...

Pour un autre encore, le choix est évident. «Je préfère garder les deux langues officielles actuelles. Je pense que pour un bon équilibre national, il est meilleur de miser sur leur neutralité ? Avec plus de volonté, elles nous éviteraient des frustrations et des tensions ».

De l'avis de deux autres informateurs, il vaut mieux maintenir les deux langues officielles. Le premier indique : «Mon choix serait de rester avec les deux langues officielles, car elles au moins ne nous sont pas intrinsèques. Au Cameroun, personne ne peut s'enorgueillir ou s'en plaindre ». Le second affirme: «Je préfère parler les deux langues officielles parce que, n'en 
déplaise au garant de la culture, elles sont adaptées aux relations internationales; avec elles, le pays est facilement ouvert à l'extérieur ».

Sur un autre registre, d'autres informateurs sont plutôt nuancés dans leurs prises de position. Pour eux, ce qui importe c'est la préservation de l'unité nationale. C'est ce qui apparait dans les propos de cet informateur qui pense qu' « entre parler une ou plusieurs langues, il n'y a qu'une seule chose à retenir. Les deux choix sont importants dans le cas où l'un ou l'autre participe à la consolidation de l'unité nationale ».

Si on s'en tient aux différents points de vue émis, adopter une ou plusieurs langues nationales c'est nier l'histoire et les préjugés que les différences culturelles ont occasionnés. Ainsi, une entreprise visant à regrouper les Camerounais autour d'une langue nationale officielle, si elle est envisagée, ne saurait être la solution adéquate aux préoccupations de l'unité nationale. De fait, le schéma actuel porté sur l'anglais et le français comme dénominateurs communs reste pertinent, car comme on pourrait le dire, ce n'est pas la différence ethnique et linguistique en soi qui pose problème, mais la gestion de cette différence.

\section{Conclusion}

La diversité ethnique et culturelle du Cameroun est un fait qui singularise ce pays parmi tant d'autres dans le monde. Elle implique, par voie de conséquence, un multilinguisme incluant plus de deux-cent-cinquante langues ethniques nationales et deux langues officielles héritées des administrations française et anglaise. Ces dernières, sur lesquelles s'est construite l'histoire qui a conduit à l'avènement du Cameroun moderne, ne semblent plus faire l'unanimité aujourd'hui. Dans un contexte où le pays entend préserver jalousement les acquis de son unité menacés par de nombreuses querelles identitaires opposant ses ethnies, et que le conflit d'envergure dans les régions anglophones du Nord-Ouest et du Sud-Ouest repose pour une part considérable sur des préoccupations en lien avec les langues, des voix demandent de repenser la question des langues officielles et en appellent à la valorisation du patrimoine linguistique national. En sourdine, et sans réel projet, des citoyens évoquent l'adoption d'une langue ou d'un corpus de quelques langues nationales pour en faire les verbes communs à tous les Camerounais. Au détour d'une réflexion dont les méandres ont transité par le recueil d'informations auprès des Camerounais de diverses appartenances ethniques, linguistiques et régionales, cet article s'est attelé à questionner la faisabilité d'une telle idée. En posant la diversité linguistique comme un corollaire de la diversité culturelle, la présente étude a procédé à l'examen des atouts et déconvenues de la multiplicité culturelle au Cameroun, avant d'interroger l'éventualité de la réduction en un dénominateur commun, le multilinguisme en place. Il résulte de cet examen qu'en ce qui concerne l'idée 
de l'unification du pays à partir de la langue, trois profils de camerounais se dégagent: les défenseurs du multiculturalisme pour qui la diversité est un atout; les adversaires de la pluralité des cultures qui y voient un péril pour la cohésion nationale ; et les modérés et surtout perplexes qui qont sont soucieux de la préservation des spécificités culturelles. Ils pensent tantôt qu'il est possible de fédérer les Camerounais autour de quelques langues en s'inspirant de la répartition du pays en ses aires culturelles, tantôt ils pensent que l'aboutissement d'un projet serait soit profitable, soit désavantageux pour le pays suivant les cas. Toutefois, il reste que la force du lien qui unit les différents groupes ethniques à leurs identités culturelles est telle que toute ambition de réduire le parler commun en une seule entité reste encore une utopie. À tort ou à raison, tout porte à croire que l'essence du Cameroun trouve son sens dans la manifestation des différences qu'il renferme.

S'il est impérieux de surpasser les clivages ethno identitaires dans un contexte où chaque langue nationale véhicule une culture, tout comme le français et l'anglais sont porteurs des systèmes d'administration hérités respectivement de la colonisation par la France et la Grande-Bretagne, le Cameroun gagnerait à mettre sur pied son propre modèle d'administration conciliant les deux approches franco-britanniques. Pour ce qui est des langues ethniques, jusqu'ici elles n'ont pas eu besoin d'être institutionnalisées à l'échelle nationale pour survivre. Les Camerounais soucieux de la survie de leurs langues ont juste besoin de les parler comme ils aiment tant parler le français et l'anglais, pour les valoriser et ainsi assurer leur pérennité. À titre d'exemple, cette réflexion préconise l'emploi des langues ethniques camerounaises dans les ménages, en famille, dans les associations, dans les temples religieux comme cela se fait de plus en plus.

\section{References:}

1. ABIA ABOA, A. L. (2012). Les langues nationales et cohésion sociale en Côte d'Ivoire. In revue d'anthropologie Nyansa-pô, 12, 721

2. GHALIOUN B. (1992). État, Nation, Ethnicité : ambivalence des concepts. Communication présentée au Colloque «Politique africaine », 11 et 12/11/1992. (Talence : IEP-CEAN)

3. HAMIDOU, N. (2007). La langue et la culture: une relation dynamique. Synergie Algérie 1, 29 - 40.

4. HENRY, A. (2008). Culture et gestion au Cameroun : le respect des rituels, source d'une entente amicale. In DAVEL E., DUPUIS J-P., et CHANLAT J-F. (Eds.) gestion en contexte interculturel : approche, 
problématiques, pratiques et plongées (pp. 1- 42). Québec, Presses de l'Université de Laval et Télé-université (UQAM).

5. LABURTHE-TOLRA P. (2010). Le fondement des problèmes d'identité en anthropologie sociale. Journal des africanistes, 77-2. Consulté le 02 mai $2020 . \quad$ URL : http://journals.openedition.org/africanistes/213.

6. M. TCHINDJANG et al. (2008). Langues et identités culturelles en Afrique. Museum International (Édition Française) doi : 10.1111/j.1755-5825.2008.00022. X.

7. MUKUM MBAKU J. (2005). Culture and Customs of Cameroon. Westport : Greenwood Press.

8. PICCARDO E. (2016). La diversité culturelle et linguistique comme ressource à la créativité. voix plurielles, 13, 57-75. $\mathrm{N}^{\circ} 1$ http://doi.org/10.26522/vp.v13i1.1370.

9. PILOTE A. MAGNAN M.-O. et VIEUX-FORT, K. (2010). Identité linguistique et poids des langues : une étude comparative entre les jeunes des milieux scolaires francophones au Nouveau-Brunswick et anglophone au Québec. Revue internationale de systémique complexe et d'études relationnelles, 6(1), 65-98.

10. SIBILLE J. (2002). La reconnaissance de la valeur culturelle des langues. Communication présentée à la Charte européenne des langues régionales ou minoritaires et la France.

11. TCHAWA P. (2012). Le Cameroun : une Afrique en miniature ? les cahiers d'Outre-mer, Journals.openedition.org/com/6640. 259. DOI : 10.4000/com.6640.

12. TCHINDA KENFO J. (2018). Les mirages de l'unité nationale au Cameroun, comment dépasser les frontières ethniques et linguistiques ? Note d'analyses sociopolitiques, 02, 1-19. 20 avril 2018, CARPADD, Montréal, pp.

13. TSOFACK, J.-B. (2002). Publicité, langue et plurivocalité au Cameroun. Sudlangues, 1, 22-36. UCAD. 